\title{
A NOTE ON GENERALIZED INVERSE FUNCTIONS
}

\author{
ROBIN HARTE
}

(Communicated by Paul S. Muhly)

\begin{abstract}
We offer the following generalization of an observation of Graham Allen about left inverses: if the elements of a semigroup of Banach algebra elements can all be given mutually commuting generalized inverses, then they can all be given invertible generalized inverses.
\end{abstract}

If $A$ is a ring, with identity 1 , we shall write $A^{-1}$ for the invertible group and

$$
A_{\text {left }}^{-1}=\{a \in A: 1 \in A a\}, \quad A_{\text {right }}^{-1}=\{a \in A: 1 \in a A\}
$$

for the larger semigroups of left and of right invertible elements, whose intersection is of course $A^{-1}$; more generally $[2,3]$ we write

$$
\bar{A}=\{a \in A: a \in a A a\}
$$

for the subset of those elements of $A$ which have generalized inverses. The commutant of a subset $K \subseteq A$ is the set

$$
\operatorname{comm}_{A}(K)=\{a \in A: a b=b a \text { for each } b \in K\} ;
$$

in particular, the centre of $A$ is given by

$$
\text { Centre }(A)=\operatorname{comm}_{A}(A) \text {. }
$$

We shall describe the ring $A$ as centre-commutative if

$$
\text { for each } a \in A \text { there is } \lambda \in \operatorname{Centre}(A) \text { for which } a-\lambda \in A^{-1} \text {. }
$$

This is familiar for example if $A$ is a real or a complex Banach algebra:

$$
\lambda \text { scalar and }|\lambda|>\|a\| \Rightarrow \exists(a-\lambda)^{-1}=-\lambda^{-1}\left(1+\sum_{n=1}^{\infty} \lambda^{-n} a^{n}\right) .
$$

In this note we generalize an observation of Hochwald and Morell [4], which was in turn stimulated by a question of Allan [1]:

THEOREM 1. If $A$ is a centre-commutative ring, if $D \subseteq A$ is a semigroup for which $A^{-1} \subseteq D \subseteq \bar{A}$, and if $g: D \rightarrow A$ is a mapping which satisfies, for each $a \in A$,

$$
a=a g(a) a
$$

and

$$
b \in A^{-1} \cap \operatorname{comm}_{A}(a) \Rightarrow g(b) g(a)=g(a) g(b),
$$

Received by the editors July 13, 1987.

1980 Mathematics Subject Classification (1985 Revision). Primary 46H05, 39B70; Secondary $47 \mathrm{D} 05$.

Key words and phrases. Generalized inverse, Banach algebra. 
then

$$
a \in D \Rightarrow a \in a A^{-1} a .
$$

ProOF. We claim that for each $a \in D$

$$
a g(a)=g(a) a:
$$

this is because if $\lambda \in \operatorname{Centre}(A)$ with $a-\lambda \in A^{-1}$, then $(a-\lambda)^{-1}$ is invertible and commutes with $a$, so that

$$
g\left((a-\lambda)^{-1}\right)=a-\lambda
$$

and

$$
g\left((a-\lambda)^{-1}\right) g(a)=g(a) g\left((a-\lambda)^{-1}\right) .
$$

From (1.5) and (1.6) we get (1.4), which now gives (1.3), since

$$
h(a)=g(a) a g(a)+(1-a g(a))
$$

is evidently an invertible generalized inverse for $a$.

The proof of Theorem 1 is exactly the argument of Hochwald and Morrel [4], who are assuming that $A$ is a Banach algebra and that $g(a)$ is a left inverse for each $a \in D$ : their conclusion is that $a$ is invertible. Allan [1] assumes that $D=A_{\text {left }}^{-1}$ and concludes that $g$ does not exist: his argument uses complex function theory and is confined to complex algebras. In [2] and [3] we called an element satisfying the condition (1.3) decomposably regular: it is rather easy to see that if a decomposably regular element is either left or right invertible then it must be invertible.

\section{REFERENCES}

1. G. R. Allan, Holomorphic left inverse functions, Proc. Conf. Banach Algebras and Several Complex Variables, Contemp. Math. vol. 32, Amer. Math. Soc., Providence, R. I., 1984.

2. R. E. Harte, Fredholm, Weyl and Browder theory, Proc. Royal Irish Acad. 85A (1986), 151176.

3. R. E. Harte, Regular boundary elements, Proc. Amer. Math. Soc. 99 (1987), 328-330.

4. S. H. Hochwald and B. B. Morrel, Some consequences of left invertibility, Proc. Amer. Math. Soc. 100 (1987), 109-110.

Department of Mathematics, UNiversity College, Cork, IRELAND 